\title{
A Medium Access Delay MAC aware Metric for Multihop Wireless Networks
}

\author{
Le Minh Duong, Véronique Vèque \\ Laboratory of Signals and Systems \\ Supélec - 3 rue Joliot-Curie \\ Gif-sur-Yvette, France \\ le-minh.duong@u-psud.fr, veronique.veque@u-psud.fr
}

\author{
Lynda Zitoune \\ Dept. of Embedded Systems - ESIEE-Paris \\ 2 boulevard Blaise Pascal-Cité Descarte \\ Noisy le Grand, France \\ zitounel@esiee.fr
}

\begin{abstract}
Internet predominant transport protocols, such as TCP and TFRC, face degradation of their performance in Multihop Wireless Networks because of their high loss and link failure rates. Many solutions have been proposed to improve the transport layer operation. These solutions are either based on network state estimation or use information from MAC layer (called MAC metrics) in a cross-layer manner to better comprehend the network state. In this paper, we define a new MAC metric called Medium Access Delay (MAD) to better reflect the network state, and provide a comparative study of MAD over other pertinent MAC metrics, their expected usage and measurement methods at MAC layer. We also investigate the behaviors of MAC metrics through several experiments in order to reveal their effectiveness in reflecting network events such as contention, collision and losse.
\end{abstract}

\section{INTRODUCTION}

In recent years, multihop wireless networks (MHWNs) have experienced an explosion of deployment due to the increasing demand for continuous connectivity regardless of the physical location. Over the past ten years, researchers gave a lot of attention in Mobile Ad hoc Networks (MANETs) which consist of wireless network adopting multihop wireless technology without deployment of wired backhaul links.In multihop wireless networks, one class acts as a relay to the cellular infrastructure whereas the other one consists of a Mesh network. Various utilizations are considered like in-building coverage, vehicular network or temporary coverage. The most widely used wireless technology for current needs is WIFI [1] standard $802.11 \mathrm{~s}$.

In the first place, these technologies were designed and deployed as extensions of the existing Internet and fixed LAN infrastructure model. Thus, many of Internet predominant protocols such as TCP and UDP are used naturally for this new kind of networks. However, there are many kind of packet losses due to wireless medium characteristics and multihop nature, such as medium access contention drops, random channel errors and route failures which should be treated properly [2].

As a consequence, these environments present a high packet loss rate at the transport layer which impairs the congestion control algorithms and tends to reduce considerably the TCP throughput [2], [3]. In recent years, a lot of solutions have been proposed to improve TCP operation on wireless links operating either at link level to recover errors or at transport level to perform loss differentiation and to use classification algorithms [4], [5], [6]. In addition, communication in wireless networks with shared medium is essentially different with that in wired networks. Nodes have to contend with each other to get access to the medium. Transport protocols like TCP usually misbehaves in MHWN by overloading the network which in turn exacerbates the contention problem. As MAC contention becomes serious, queueing delay, backoff and transmission delays and collision losses increase while the throughput decreases. Hence, congestion control mechanisms at transport layer should be aware of MAC layer events (contention/collision, losses) to keep the network load at a reasonable level. This approach is qualified of "cross-layer" [7] where the layered protocols are not designed independently but in a combined manner. Note that some proposed approaches are based on network state estimation and in our opinion, are not actual cross-layer ones. In order to improve the transport service, we think that it is very important to investigate the relationship between the contention or congestion states and information from MAC layer, called MAC metrics. Like in routing improvement using metrics [8], we propose a new MAC metric called MAD (Medium Access Delay) to reflect accurately MAC states, i.e contention, collision and losses, and compare it to some pertinent MAC metrics. We then investigate the behavior of all these MAC metrics through several simulation experiments in order to reveal their effectiveness in reflecting network states.

The paper is organized as follows. The next section gives a brief review of related works. Section III provides the definition of both the new metric MAD and the pertinent MAC metrics that we assess in our study. After the description of simulation scenarios in Section IV, we comment the simulation results that exhibit the effectiveness of the MAC metrics. Finally, we conclude the paper in Section VI.

\section{RELATED WORKS}

Since end-to-end information is not enough to solve the problems in MHWNs, most of proposed schemes have a common ground that they try to take advantage of the MAC layer information to have better knowledge about what happens at lower layers. The exploited MAC information forms the 
MAC metrics, each of which is a collection of one or more parts taken from the DCF (Distributed Coordination Function) scheme and, they may fall into following categories: packet delay, medium busyness, MAC throughput, transmission and retransmission attempt numbers. MAC metrics are then sent upward to transport layer and are used in various ways to improve the transport protocols.

Cross layer design of MHWN to improve TCP performance has been first introduced in [9] to make the ad hoc routing function notifying the transport protocol of link failures. [10] proposes a mechanism which enables TFRC to estimate the optimal network load level by considering the MAC layer contention. An optimum round-trip time is computed from both backoff and transmission delays at MAC layer collected from all hops from source to destination. The current RTT is then compared to this optimum value to estimate the contention level and to accordingly adjust the traffic rate. To obtain the channel utilization information, [5] collects the Channel Busyness ratio computed at each node and then estimates the network available bandwidth. The estimated value is then attached to every packet so that it can reach to the destination. This information is then used to adjust the traffic pumped into the network. [6] uses the channel busyness ratio and effective throughput computed at each node to assess the current network capacity in terms of both channel utilization and collision level. To derive contention level along the packet path, [11] has proposed to calculate periodically the MAC service time from all hops along the path. The destination compares the total MAC service time and the throughput of two consecutive intervals to determine whether the source should increase or decrease its rate.

From these works, we note MAC metrics was used to improve the transport protocols but without any comparisons between them. So, we claim that it is very important to study the effectiveness of the MAC metrics, i.e., their ability to reflect the problems of lower layer network operation. The question is that comparing these metrics, which one is better in reflecting network events such as BER loss, collision loss, congestion loss, link failure or network states such as collision level, contention level and medium busyness. We proceed in a systematic way by simulating various network situations and measuring the MAC metrics in order to answer the question of effectiveness.

\section{MAC METRICS}

\section{A. 802.11 DCF model overview}

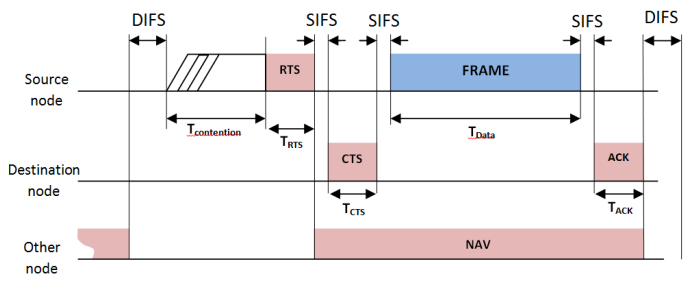

Fig. 1: IEEE 802.11 basic medium access mechanism and data delivery procedure
DCF [1], the mainly used medium access scheme of IEEE 802.11 standard, aims at minimizing collisions when sending a packet. DCF uses both backoff process that determines the packet service time [12] and ARQ mechanism to enable reliability at MAC.

Refer to Figure 1, two main parameters have to be defined, $T_{s u c}$ and $T_{c o l}$, which respectively represent the average time period associated with one successful transmission and the average time period associated with collisions [5] and are computed by:

$$
\begin{aligned}
T_{\text {suc }} & =T_{\text {rts }}+T_{\text {cts }}+T_{\text {data }}+T_{\text {ack }}+3 * T_{\text {sifs }}+T_{\text {difs }} \\
T_{\text {col }} & =T_{\text {rts }}+T_{\text {sifs }}+T_{\text {cts }}+T_{\text {difs }}
\end{aligned}
$$

or if RTS/CTS mechanism is not used:

$$
\begin{gathered}
T_{\text {suc }}=T_{\text {data }}+T_{\text {ack }}+T_{\text {sifs }}+T_{\text {difs }} \\
T_{\text {col }}=T_{\text {data }}+T_{\text {ack_timeout }}+T_{\text {difs }}
\end{gathered}
$$

In the following, we introduce the definition of some interesting metrics computed using $T_{\text {suc }}$ and $T_{c o l}$. These metrics are rather simple to compute since IEEE 802.11 MAC provides several facilities to obtain the necessary values.

\section{B. The Average Transmission Attempt}

The Average Transmission Attempt [13], AT A, is defined as the fraction of total transmission and retransmission attempts that the MAC carries out to the total number of successfully transmitted packets in an interval, as defined in equation 3.

$$
A T A=\frac{\sum N_{a t}^{i}}{N_{s p}}
$$

where $N_{s p}$ is the number of successfully transmitted packets and $N_{a t}^{i}$ is the number of attempts that the MAC takes to transmit a packet $i$ until it receives MACK or drops the packet. Thus, $A T A$ is relatively sensitive to collision level around a node.

\section{The Average Transmission Time}

The definition of the Average Transmission Time ATT is derived from the MAC Service Time $T_{s r v}$ one. ATT is the average MAC service time of a successfully transmitted packet in an interval. To calculate $A T T$, the sum of service times of every packet arrived at MAC during an interval is made and then is divided by the total number of transmitted packets whose MACKs are received successfully in that interval [13].

$$
A T T=\frac{\sum T_{s r v}}{N_{s p}}
$$

where $N_{s p}$ is the number of successfully transmitted packets. The $A T T$, by this definition, comprises the backoff delay and transmission delay and therefore can be used to indicate the contention level around a node. If the number of neighboring nodes which have traffic to transfer over the channel increases, a node has to defer longer in backoff stage to access the medium and may have higher probability of packet collision which in turn introduces longer transmission delay. $A T T$ is sensitive to offered load at MAC and collision level in node's neighborhood. 


\section{The Medium Access Delay}

The Medium Access Delay, $M A D$, is simply defined as the average total backoff delay for a packet at MAC layer before it is successfully transmitted or dropped after several failed retransmissions in an interval. By this definition, $M A D$ includes the backoff duration at the first time it enters MAC layer and all other backoff periods it has to defer after each failed (re)transmission and NAV delay in each backoff stage.

$$
M A D=\frac{\sum^{N_{a p}} \sum T_{\text {contention }}^{i}}{N_{a p}}
$$

where $N_{a p}$ is the number of arrival packets in the interval and $T_{\text {backoff }}^{i}$ is the backoff time at the $i^{\text {th }}$ transmission attempt (Fig. 1). Note that maximum retransmission number is limited by the value RetryLimit defined in IEEE 802.11 MAC.

If the value of $M A D$ increases, either or both two possibilities may rise. First, the channel is more busy so that the node has to defer longer to have a transmission opportunity. Second, the number of retransmissions increases due to higher level of collision with a note that the node returns to backoff stage after each failed transmission. Therefore, $M A D$ may be used to indicate both the medium busyness and collision level around a node.

\section{E. The Channel Busyness Ratio}

The Channel Busyness Ratio $R_{b}$ is defined in [5] as the ratio of total busy periods of successful transmission or collision to the duration of observed time interval ( $\left.T_{\text {interval }}\right)$.

$$
R_{b}=\frac{\sum T_{\text {suc }}+\sum T_{c o l}}{T_{\text {interval }}}
$$

where $T_{s u c}$ and $T_{c o l}$ are defined by equations (1) and (2). If $R_{b}$ is high, it means that the shared channel is used more frequently by the nodes around with the increase of offered load. Zhai et al. [5] claim that if the collision probability is smaller than 0.1 , there is an optimal point of $R_{b}$ for the operation of the network where the throughput is maximized and, delay and delay variation are small. At that point, $R_{b}$ is around $0.90 \sim 0.95$. Therefore, [5] uses $R_{b}$ to calculate the available bandwidth of the network and to adjust the traffic rate accordingly.

However, using only $R_{b}$ to estimate channel capacity of a link may be not sufficient since [5] ignores the hidden terminal problem where packets may collide at a high probability, leading to the decrease of channel utilization [6].

\section{F. The Effective MAC Throughput}

The Effective MAC Throughput EMT is the fraction of the total number of successfully transmitted packets to the total MAC service time of packets arrivals in an interval (as in equation 7).

$$
E M T=\frac{\sum_{s p}^{N_{s p}} S}{\sum T_{s r v}}
$$

where $T_{s r v}$ is the time interval from the instant a frame starts to contend for transmission to the instant the transmitter receives correctly the MACK of that frame or drops it after several failed retransmissions [14]. $S$ is the packet size with assumption that all packets have the same size.

Note that the two components of EMT are inversely proportional to each other. Indeed, in the same observed time with assumption that the node always has packet to send, if the number of successfully transmitted packets increases, the service time spent for a packet (in average) at MAC decreases and vice versa. This makes EMT sensitive to MAC losses which are largely caused by collision between sending nodes which share the same channel.

\section{Simulation SCEnarios}

As pointed out in the introduction, our objective is to show that the metric $M A D$ is very effective to represent the network behavior compared to other MAC metrics cited earlier. As a consequence, MAD can be used by transport layer to refine its control operation. So, our aim is to evaluate the previous MAC metrics (of the section ) in both saturated and non saturated network. In the saturated case, the network suffers from congestion, collision and packets in error, our idea is to evaluate the close coupling between the metric $M A D$ and congestion/contention level.

We have specified two main scenarios. Scenario (1) investigates the increase of traffic load induced by either (1.1) the increase of one source bit rate, and (1.2) several parallel connections causing interferences among each other.

Scenario 2 is built to assess the impact of different channel BER on MAC metrics. The BER of the channel changes such as in $\{0,10 \mathrm{e}-6,10 \mathrm{e}-4,10 \mathrm{e}-3\}$.

The same simulation topology is used for scenarios (1) and (2). Except for scenario (1.2), we use a chain topology with 9 hops, each pair of nodes being $200 \mathrm{~m}$ far from each other. The traffic load is generated by one CBR source and is transmitted over connections established along the topology, from node 0 to node 9. In the experiments, CBR packet size is fixed to 1000 bytes while the CBR rate increases after each simulation from 0.5 to $2 M b p s$.

For scenario (1.2), the considered topology is showed in figure 2 . We set up 3 connections to carry 3 CBR flows: the first one from node 0 to node 9 with $1 \mathrm{Mbps}$, the second one from node 10 to node 12 with $0.5 \mathrm{Mbps}$ rate and the last one from node 14 to node 13 with $0.5 \mathrm{Mbps}$ rate.

In this work, we used the 802.11 MAC model of the $n s-2$ simulator (version 2.34) http://isi.edu/nsnam/ns. The table I displays the general configuration parameters we used in the simulations.

TABLE I: General configuration for each Scenario

\begin{tabular}{|c|c|}
\hline Parameters & Value \\
\hline Propagation Model & TwoRayGround \\
\hline MAC protocol & $802.11 \mathrm{a}$ \\
\hline Channel Capacity & $6 \mathrm{Mbps}$ \\
\hline Interface queue size & 50 \\
\hline Carrier Sensing Range & $\simeq 500 \mathrm{~m}$ \\
\hline Transmission Range & $\simeq 250 \mathrm{~m}$ \\
\hline CBR packet size & 1000 bytes \\
\hline
\end{tabular}




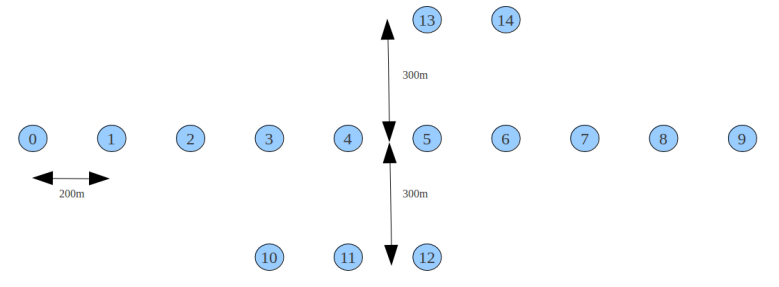

Fig. 2: Parallel topology for scenario 1.2

\section{RESUlTS AND DISCUSSION}

There are two observed states of the network operation: nonsaturated and saturated. In non-saturated state (CBR bit rate $\leq 1.18 \mathrm{Mbps}$ ), there is no loss due to collision and congestion since the collisions are very rare. The MAC metrics display representative behaviors for some network events. When the rate reaches a threshold about $1.18 \mathrm{Mbps}$, the network enters into the saturated state where the collisions become more frequent and losses happen. In the following and due to space constraints, we only show up some remarkable results of the study.

\section{A. Average Transmission Attempt}

In non-saturated state, the metric is equal to its intrinsic value i.e 1 for all nodes and regardless of traffic rate, packet size and node's position (Fig. 3.a). This means that the MAC protocol performs only one attempt to successfully transmit a packet. Moreover, $A T A$ is sensitive to channel error losses as it exceeds its intrinsic value at the node where losses occur (Fig. 3.c). This is because the node needs more than one attempt to transmit a packet or at worst, it has to drop it after reaching the maximum number of attempts.

In saturated state, $A T A$ value depends on the node's position and on the traffic load. It exceeds 1 in the first nodes where the contention is the highest while at the ending nodes $A T A$ is close to 1 (Fig. 3.a and b). However these variations are almost unremarkable compared to those of other metrics. In conclusion, whatever the MAC state, ATT variation is relatively flat compared to $A T T$ and $M A D$. As a consequence we think that this metric is not effective and can not be used to improve the transport protocol operation.

\section{B. Average Medium Access Delay}

In non-saturated state, $M A D$ value is constant and very low (Fig 4.a). Because the MAC layer invokes only one backoff stage, the $M A D$ 's value is the backoff time with the minimum contention window of the first transmission attempt. $M A D$ in this case is also independent of packet size, node's position and traffic rate as long as it is smaller than the rate threshold. When the traffic load increases as in Fig 4.a and b, $M A D$ reflects well medium busyness and collision level in the neighborhood of a node. When channel losses occur (Fig. 4.c), the number of attempts to transmit a packet increases as well as the number of backoff stages thanks to ARQ mechanism. After each failed transmission, the backoff time is longer due to the increment of MAC contention window [1]. Hence, $M A D$ as the total number of all backoff times calculated in the interval increases as well. After the loss, $M A D$ is back to normal value. Thus,
$M A D$ can also be used to indicate channel loss along the path in non-saturated condition.

The $M A D$ value depends on node position, network load and loss error, so $M A D$ provide an accurate indication on the MAC state. As $M A D$ metric is only related to the channel access, it is closely coupled with contention level and losses, so $M A D$ allows to detect them earlier. This is a very interesting feature when controlling the transmission of multimedia applications.

\section{Average Transmission Time}

In the non-saturated network, $A T T$ behaves like $A T A$ as shown in figure (Fig. 5.c) in case of BER losses. The MAC protocol needs only one attempt to transmit successfully a packet. As the backoff time is rather small, ATT is still constant for given packet size since it includes the transmission time (Fig. 5.a). This is because when there is no collision loss, $A T T$ value depends also on packet size since it includes the transmission time. In saturated state $A T T$ values increase, and like $M A D$, they depend on the node's position, traffic rate and packet size. Fig. 5.a and b also show that $A T T$ is sensitive to traffic load in terms of either the data rate or the number of concurrent connections.

If the traffic rate is rather high, the losses force the network to enter into the saturated state. The reason is that when channel error occurs, the sending node needs more time to send a frame due to retransmissions, it makes ATT increase. After the loss, if the traffic rate is low enough, i.e., the arrival rate is smaller than the frame service rate at MAC, the average queue length is always smaller than 1 and the next frame does not almost need to contend for the medium and ATT value varies around a "steady" value. In contrast, if the traffic rate is high, the average queue length of nodes in the neighborhood is greater than 1 after the loss, then the sending node has to contend for the medium with its neighbors. Therefore, collision occurs leading to overload the network.

As for $M A D, A T T$ reflect faithfully the MAC states, but in addition to the access time, it includes the successful transmission delays. We think that if the transport control protocol has to react quickly to contention, we must use $M A D$ rather than $A T T$.

\section{Channel busyness Ratio}

Figure 6 displays the average value of $R_{b}$ of nodes with several traffic rates. We observe that as long as the traffic rate is smaller than the threshold ( $\simeq 1.18 \mathrm{Mbps}$ ), $R_{b}$ of each node is rather stable and depends on the node's position. The higher the rate is, the higher the values of $R_{b}$ are. At some nodes, $R_{b}$ is around $96 \%$ when the traffic rate approaches the threshold. However, when the rate exceeds the threshold, $R_{b}$ becomes variable and its average decreases sharply at some nodes. This is because when the rate does not overload the network, the channel time is spent for successful transmission attempts and backoff stage, the collision time is almost zero and the transmission time is almost constant. If the rate increases but still does not overload the network, the channel time used 


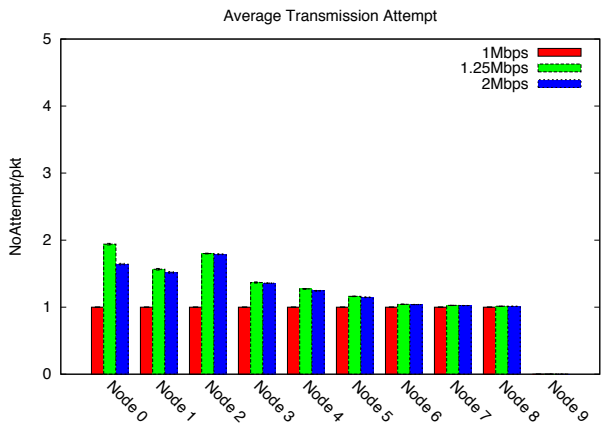

(a) Scenario 1.1 with traffic rate variation

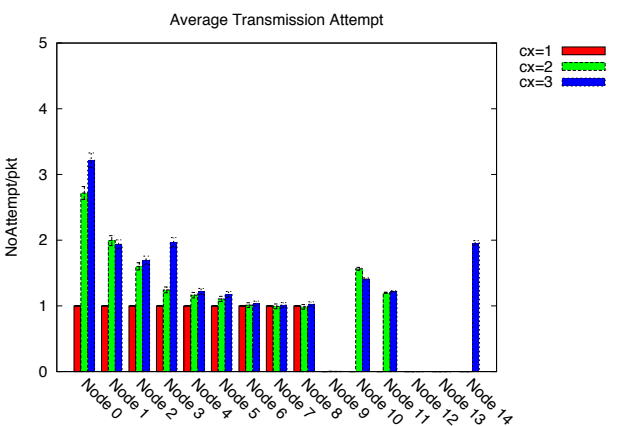

(b) Scenario 1.2 with connection variation

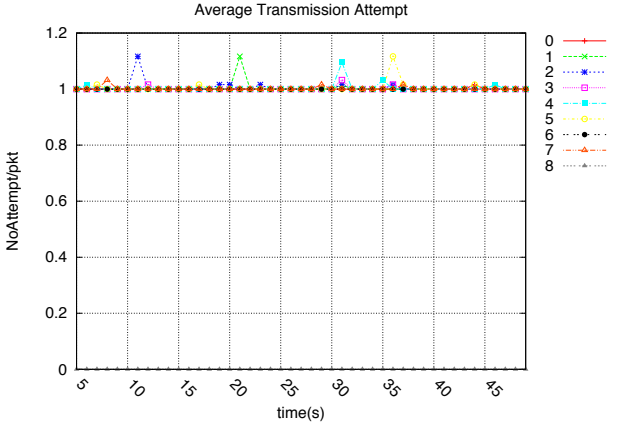

(c) Scenario 2 with 1 connection, rate $=0.5 \mathrm{Mbps}$ and $\mathrm{BER}=10 \mathrm{e}-6$

Fig. 3: Results for Average Transmission Attempt $A T A$

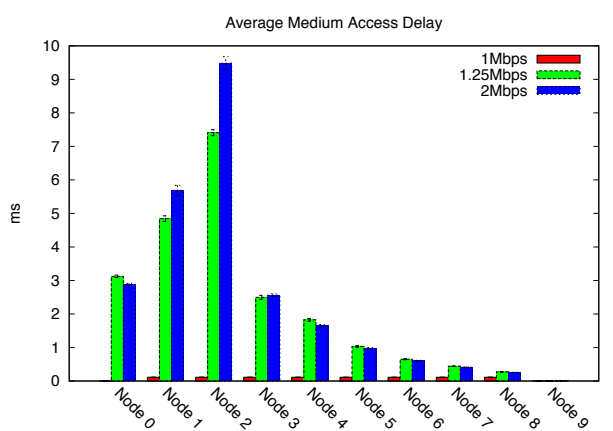

(a) Scenario 1.1 with traffic rate variation

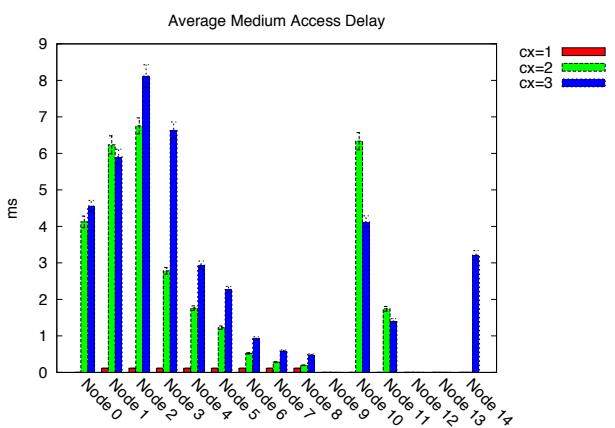

(b) Scenario 1.2 with connection variation

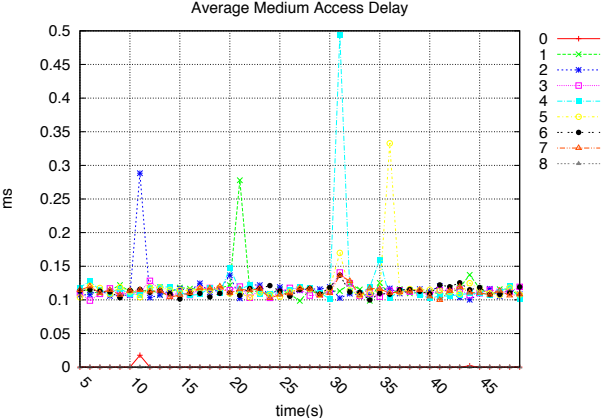

(c) Scenario 2 with 1 connection, rate $=0.5 \mathrm{Mbps}$ and $\mathrm{BER}=10 \mathrm{e}-6$

Fig. 4: Results for Average Medium Access Delay $M A D$

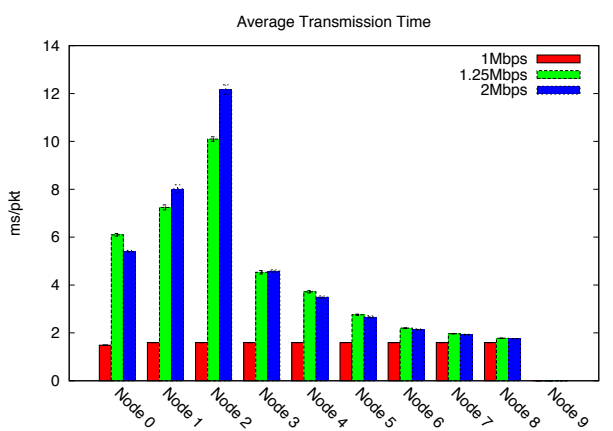

(a) Scenario 1.1 with traffic rate variation

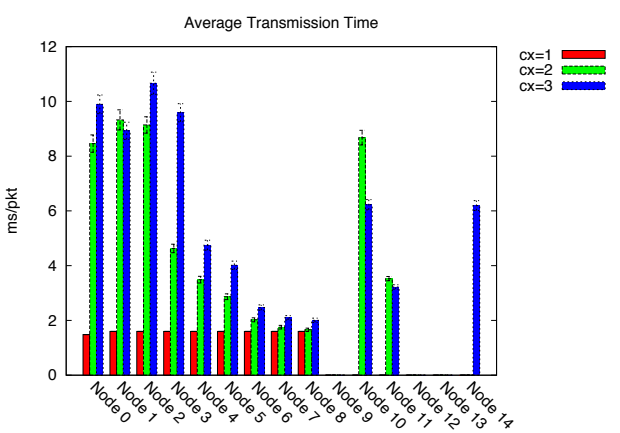

(b) Scenario 1.2 with connection variation

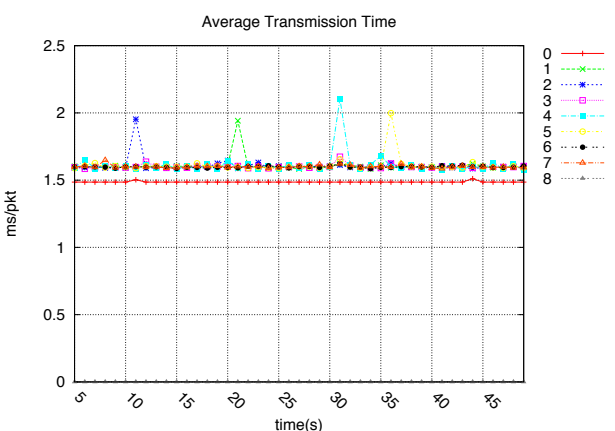

(c) Scenario 2 with 1 connection, rate $=0.5 \mathrm{Mbps}$ and $\mathrm{BER}=10 \mathrm{e}-6$

Fig. 5: Results for Average Transmission Time ATT

for successful transmission increases as well while the idle time decreases, thus $R_{b}$ increases. But when the traffic rate overloads the network, collision and losses happen. Therefore, nodes have to spend more time to contend for the channel making the most part of channel time is used for backoff and $R_{b}$ decreases as the consequence.

\section{E. Effective MAC Throughput}

The obtained results are expected since the throughput is inversely related to $M A D$ or $A T T$ delays. In agreement with the work of [14], EMT of each node reaches its maximum value under non-saturated state, regardless of traffic load (under the threshold) and node's position (Fig. 7). In non-saturated state, EMT is also sensitive to channel error at the node where losses occur (Fig. 8). When contention occurs, EMT decreases sharply to a local peak value and then becomes variable (Fig. 8). Transport protocols may observe $E M T$ of nodes to react properly to the interference change along the path.

From the simulation results, we conclude that $A T A$ metric is not enough sensitive to MAC state variation. So, we can not consider it as an effective metric. Also, we note that $M A D$ and $A T T$ reflect faithfully the MAC states compared to $R_{b}$, since they point out nodes where the contention or the collision occur. As a conclusion, we propose to use $M A D$ as the effective MAC metric as it permit to signal problems 


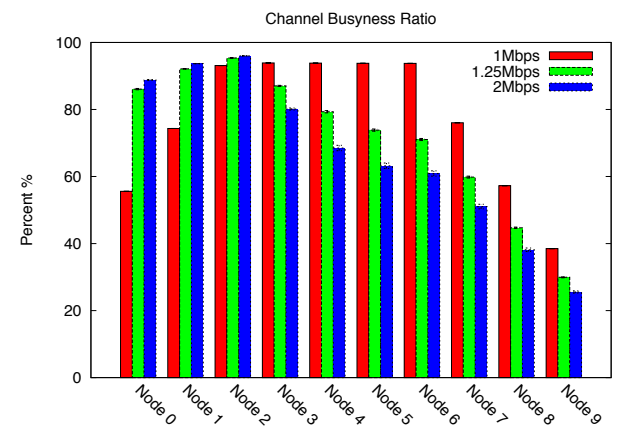

Fig. 6: Results for Channel Busyness Ratio $R_{b}$

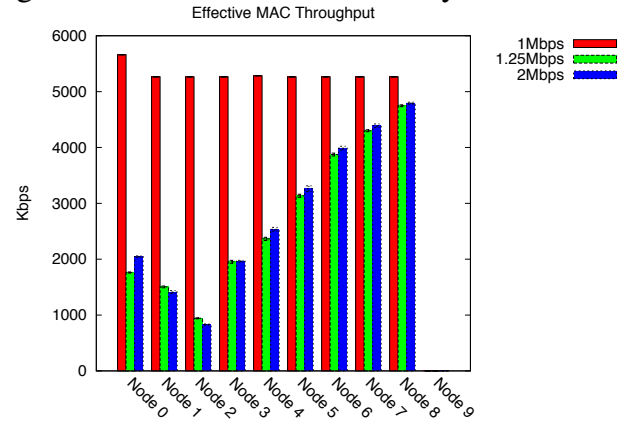

Fig. 7: Results for Effective Throughput EMT, scenario 1.1

(contention and packet losses) earlier than $A T T$.

\section{CONCLUSION}

Transport protocols have several problems working in $\mathrm{MH}-$ WNs. Using metrics from MAC layer to improve their performance is a popular research direction. The transport protocol may adapt the packet size and its sending rate by observing the evolution of the metrics to achieve high network performance.

In this study, we proposed a new MAC metric named $M A D$ and compared it to the well-known MAC metrics through various scenarios to show the effectiveness of each one to reflect the network behavior.

Except $A T A$, all the metrics invoked in this study react to network state variation such as contention and losses, and can be used to indicate the network operation mode: saturated and non-saturated.

$A T T$ and $M A D$ introduce a better feature that their values in non-saturated network are independent of node number, position, packet size and traffic rate (as long as it is smaller than a threshold). Moreover in the saturated state, these metrics can also be used to differentiate loss reasons or to indicate medium busyness, contention and collision level along traffic path. We propose to use $M A D$ as the effective MAC metric since it gives an earlier indication of contention and packet losses compared to $A T T$. We think that $M A D$ metric is more appropriate to control the transmission of multimedia applications over MHWNs.

Our future work will concentrate on making use of $M A D$ metric to improve the operation of some transport protocols such as TFRC or DCCP in MHWNs.

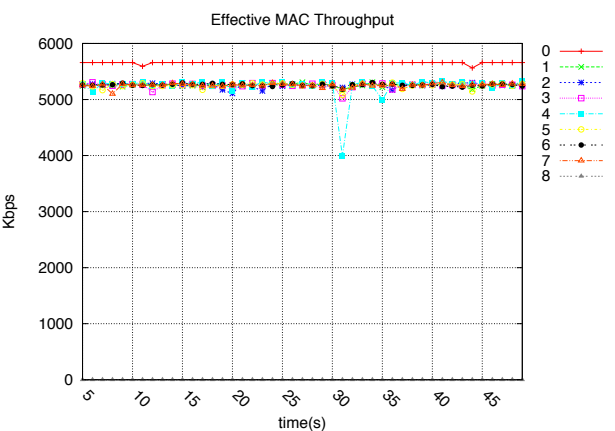

Fig. 8: Results for Effective Throughput EMT, scenario 2

\section{REFERENCES}

[1] "IEEE 802.11-2007, Wireless LAN Medium Access Control (MAC) and Physical Layer (PHY) Specifications," June 2007.

[2] Z. Fu, X. Meng, and S. Lu, "How Bad TCP Can Perform In Mobile Ad Hoc Networks," in Proceedings of IEEE ISCC 2000, 2002, pp. 298-303.

[3] Z. Fu, P. Zerfos, H. Luo, S. Lu, L. Zhang, and M. Gerla, "The impact of multihop wireless channel on tcp throughput and loss," 2003, pp. $1744-1753$.

[4] A. Seddik-Ghaleb, Y. Ghamri-Doudane, and S. Senouci, "Effect of Ad Hoc Routing Protocols on TCP Performance within MANETs," in [Invited paper] IWWAN 2006, 2006.

[5] H. Zhai, X. Chen, and Y. Fang, "Improving transport layer performance in multihop ad hoc networks by exploiting MAC layer information," IEEE Transactions on Wireless Communications, vol. 6, no. 5, pp. 16921701, 2007.

[6] P. Navaratnam, H. Cruickshank, and R. Tafazolli, "A link adaptive transport protocol for multimedia streaming applications in multi hop wireless networks," in MobiMedia '07: Proc. of the 3rd int. conf. on Mobile multimedia communications, 2007, pp. 1-6.

[7] V. Srivastava and M. Motani, "Cross-layer design: a survey and the road ahead," IEEE Communications Magazine, vol. 43, no. 12, pp. 112-119, Dec. 2005. [Online]. Available: http://dx.doi.org/10.1109/ MCOM.2005.1561928

[8] S. M. Das, H. Pucha, K. Papagiannaki, and Y. C. Hu, "Studying wireless routing link metric dynamics," in Proceedings of the 7th ACM SIGCOMM conference on Internet measurement, ser. IMC '07, 2007, pp. 327-332.

[9] G. Holland and N. Vaidya, "Analysis of TCP performance over mobile ad hoc networks," in Proceedings of the 5th annual ACM/IEEE international conference on Mobile computing and networking, ser. MobiCom '99, 1999, pp. 219-230.

[10] M. Li, C. soo Lee, E. Agu, M. Claypool, and R. Kinicki, "Performance Enhancement of TFRC in Wireless Ad Hoc Networks," in In DMS 04: Proceedings of the 10th International Conference on Distributed Multimedia Systems, 2004.

[11] E. Hamadani and V. Rakocevic, "A cross layer solution to address TCP intra-flow performance degaradation in multihop ad hoc networks," Journal of Internet Engineering, vol. 2, 2008.

[12] K. Wang, F. Yang, Q. Zhang, and Y. Xu, "Modeling path capacity in multi-hop ieee 802.11 networks for qos services," IEEE Trans. Wireless Commun, vol. 6, 2007.

[13] S. Yan-jing, L. Xue, and Z. Bei-bei, "An available transmission time routing metric for wireless ad-hoc sensor networks," International Journal of Distributed Sensor Networks, vol. 2011, no. 815987, p. 6, 2011.

[14] H. Zhai, X. Chen, and Y. Fang, "How well can the ieee 802.11 wireless lan support quality of service?" IEEE Transaction on Wireless Communications, vol. 4, pp. 3084-3094, 2005. 